\title{
Triorchidism: Genetic and imaging evaluation in an adult male
}

\author{
Arben Belba ${ }^{1}$, Valentina Riversi ${ }^{2}$, Francesca Mari $^{3}$, Eleonora Cellesi ${ }^{1}$, Roberto Ponchietti ${ }^{1}$ \\ ${ }^{1}$ Urological and Andrological Unit, Department of Medicine, Surgery and Neuroscience, Siena, Italy; \\ 2 Imaging Department AOUS of Siena, Italy; \\ ${ }^{3}$ Medical Genetics Unit, Department of Medical Biotechnologies, Siena, Italy.
}

\begin{abstract}
Summary
We report the results of imaging and cytogenetic studies in a case of triorchidism in a 54 years old male without any associated anomaly.

A scrotal ultrasonography revealed the presence of two testes within the left hemiscrotum with complete septation and echotexture and vascular flow pattern similar to the vascular flow of the normal right testis.

There was no focal abnormal echogenicity suggesting malignancy. Scrotal MRI confirmed two soft-tissue structures in the left hemiscrotum with normal signal intensity at T1w and T2w images. Both testes had a tunica albuginea with low-signal intensity. Cytogenetic analysis resulted in normal male karyotype 46XY. Array-CGH analysis detected the presence of two interstitial rearrangements: $a \sim 120 \mathrm{~Kb}$ deletion of chromosome 1 and $a \sim 140 \mathrm{~Kb}$ deletion of chromosome 16. Currently there are little details on the functions of both genes.
\end{abstract}

KEY WORDS: Polyorchidism; Ultrasonography; MRI; Cytogenetic evaluation.

Submitted 4 January 2014; Accepted 31 January 2014

\section{INTRODUCTION}

Polyorchidism is a rare genital anomaly defined by the presence of supernumerary testes usually within the scrotum. To date there have been almost 200 cases reported in the literature $(1,2)$. The most common presentation of polyorchidism is triorchidism with the supernumerary testis being confined to the left side. The exact mechanism for occurence of polyorchidism is still unknown. Several theories have been proposed, including peritoneal folding, segmentation of the primitive gonads, longitudinal or transverse division of the genital ridge. No single theory can explain all types of polyorchidism since some involve testicular tissue only and others involve complete duplication of the testis, epididymis and vas deferens $(3,4)$. Most cases of polyorchidism are found incidentally in association with undescended testis, hydrocele, hernia or torsion. It is also reported as increased risk of testicular malignancy.

No conflict of interest declared.

\section{Case report}

A 54 years old man presented to our outpatients Department with complaints of erectile dysfunction. His past medical history revealed diabetes type 1 since the age of 28 years and 10-year history of a left-sided scrotal swelling associated with some discomfort. He was married and fathered two daughters.

Abdominal examination was normal with no palpable mass or groin herniae. Scrotal examination revealed a normal right testis and scrotal content, but on the left side there were two similar size lumps. Laboratory studies, including hormonal and oncological markers, were within normal limits.

A scrotal ultrasonography revealed the presence of 2 testes within the left hemiscrotum with complete septation and echotexture and vascular flow pattern similar to the vascular flow of the normal right testis. There was no focal abnormal echogenicity suggesting malignancy (Figure 1). Scrotal MRI confirmed 2 soft-tissue structures in the left hemiscrotum with normal signal intensity at T1w and T2w images. Both testes had a tunica albuginea with low-signal intensity (Figure 2).

Chromosomal preparations for the karyotype analysis were obtained according to standard techniques. Cytogenetic analysis at a resolution of 400 bands resulted in normal male karyotype 46XY.

Patient DNA was analysed by Array-CGH analysis using a commercially available oligonucleotide microarrays containing about 44.000 60-mer probes (Human Genome CGH Microarray 44B Kit, Agilent Technologies, Santa Clara, California) according to the manufacturer's instructions.

Array-CGH analysis detected the presence of two interstitial rearrangements: a $\sim 120 \mathrm{~Kb}$ deletion of chromosome 1 (arrlq31.1 $(79,356,819-79,476,571) \times 1)$ and $\mathrm{a} \sim 140 \mathrm{~Kb}$ deletion of chromosome 16 (arr 16q22.1(70,052,16470,193,889)x1). Parents were not available for testing. The microdeletion of chromosome 1 includes the ELTD 1 (latrophilin and seven transmembrane domain containingl) gene, while the microdeletion of chromosome 16 includes the PDPR (Pyruvate Dehydrogenase Phosphatase Regulatory Subunit) gene. Currently there are little details on the functions of both genes. The protein encoded by ELTD1 could be involved in cardiac development. The 
Figure 1.

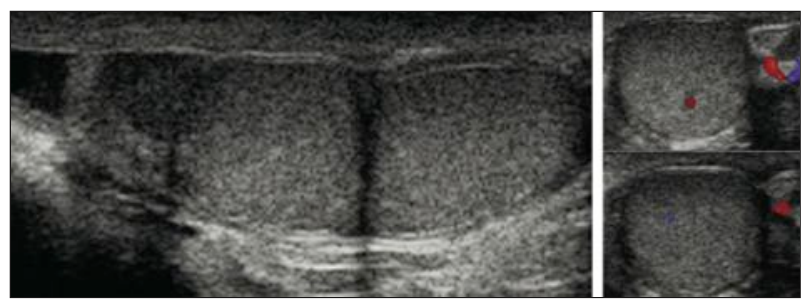

A. Sagittal sonographic image of the left scrotum showing two testicles completely separated. The supernumerary testis is smaller and superior to the more normal-sized and it appears as an oval, isoechoic mass with a homogeneously echogenic pattern identical to that of the other testicle; they share the epididymis and the vas deferens.
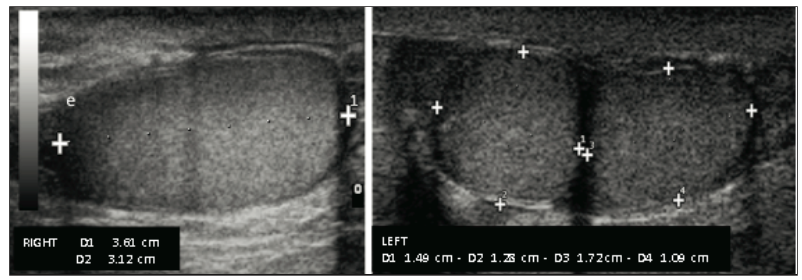

B. Color Doppler image showing the same vascular pattern of the normal and accessory testicle.

c. Both testicles have approximately the same size.

protein encoded by PDPR is a regulatory subunit of human mitochondrial pyruvate dehydrogenase phosphatase. It decreases the sensitivity of PDP1 to magnesium ions, and this inhibition is reversed by the polyamine spermine. Both these proteins are expressed also in testis.

A diagnosis of triorchidism was made and the patient was placed in sonographic follow up.

Figures are reported in Supplementary materials posted on www.aiua.it

\section{Discussion}

Polyorchidism is a rare congenital anomaly defined by the presence of more than two histologically proven testes. The commonest variant is triorchidism, the supernumerary testis being commonly reported on the left side which often appears as a painless scrotal mass or may be found as an incidental finding on sonography. Ultrasonography is diagnostic, MRI plays a confirmatory role and may provide additional information in conditions that may complicate polyorchidism, such as torsion, cryptorchidism and neoplasia (4-6).

Most patients with polyorchidism have a normal 46XY karyotype and adult patients have normal secondary sexual characteristics. Because polyorchidism is rare and poorly described, genetic studies are lacking.

Chromosomal abnormality such deletion of the long arm of chromosome 21 has been reported in a severe malformed male newborn (7).

The current knowledge does not allow to attribute a causative role to the aploinsufficency of two genes in determining the phenotype of our patient. Additional Array-CGH analysis in patients with supernumerary testis are required to increase data and to define the role of these genes in the pathogenesis of polyorchidism.

Management of polyorchidism has been the subject of much debate (8). The incidence of testicular malignancy in polyorchidism is between $5.7-7 \%$ and was found only in a non-scrotal (abdominal or inguinal) supernumerary testis. With recent improvements in imaging techniques such as ultrasound and MRI scans, most cases of polyorchidismc can be diagnosed and followed up accurately without any need for surgical exploration or histological examination. Conservative treatment with sonographic follow-up is the choice of treatment in uncomplicated cases (9).

\section{REFERENCES}

1. Bergholz R, Wenke K, Polyorchidism: A Meta-Analysis. J Urol. 2009; 182:2422-2427.

2. Savas M, Yeni E, Ciftci H, et al. Polyorchidism: a three-case report and review of the literature Andrologia 2010; 42:57-61.

3. Thum G, Polyorchidism: case report and review of literature. J Urol. 1991; 145:370-372.

4. Singer BR, Donaldson JG, Jackson DS. Polyorchidism: functional classification and management strategy. Urology. 1992; 39:384-388.

5. Chung TJ, Yao WJ. Sonographic features of polyorchidism. J Clin Ultrasound. 2002; 30:106-108.

6. Yalçınkaya S, Sahin C, Sahin AF. Polyorchidism: sonographic and magnetic resonance imaging findings. Can Urol Assoc J. 2011; 5:84-86

7. Arslanoglu A, Tuncel SA, Hamarat M. Polyorchidism: color Doppler ultrasonography and magnetic resonance imaging findings. Clin Imaging. 2013; 37:189-191.

8. Shabtai F, Schwartz A, Hart J, et al. Chromosomal anomaly and malformation syndrome with abdominal polyorchidism. J Urol. $1991 ; 146: 833-834$.

9. Nayak SP, Sreejayan MP. Management of supernumerary testis in an adult: case report and review. Andrologia 2011; 43:149-152.

\section{Correspondence}

Roberto Ponchietti, MD (Corresponding Author)

roberto.ponchietti@unisi.it

Eleonora Cellesi, MD

Arben Belba, MD

Urological and Andrological Unit, Department of Medicine,

Surgery and Neuroscience, University of Siena, Siena, Italy

Valentina Riversi, $\mathrm{MD}$

Imaging Department AOUS of Siena, Siena, Italy

Francesca Mari, MD PhD

Medical Genetics Unit, Department of Medical Biotechnologies, Siena, Italy 